ISSN 1855-3966 (printed edn.), ISSN 1855-3974 (electronic edn.)

ARS MATHEMATICA CONTEMPORANEA 22 (2022) \#P3.05

https://doi.org/10.26493/1855-3974.2645.8fc

(Also available at http://amc-journal.eu)

\title{
On the essential annihilating-ideal graph of commutative rings*
}

\author{
Mohd Nazim (D), Nadeem ur Rehman ${ }^{\dagger}$ \\ Department of Mathematics, Aligarh Muslim University, Aligarh-202002, India
}

Received 30 May 2021, accepted 8 September 2021, published online 9 June 2022

\begin{abstract}
Let $R$ be a commutative ring with unity, $A(R)$ be the set of annihilating-ideals of $R$ and $A^{*}(R)=A(R) \backslash\{0\}$. In this paper, we introduced and studied the essential annihilatingideal graph of $R$, denoted by $\mathcal{E} \mathcal{G}(R)$, with vertex set $A^{*}(R)$ and two distinct vertices $I_{1}$ and $I_{2}$ are adjacent if and only if $\operatorname{Ann}\left(I_{1} I_{2}\right)$ is an essential ideal of $R$. We prove that $\mathcal{E} \mathcal{G}(R)$ is a connected graph with diameter at most three and girth at most four if $\mathcal{E} \mathcal{G}(R)$ contains a cycle. Furthermore, the rings $R$ are characterized for which $\mathcal{E} \mathcal{G}(R)$ is a star or a complete graph. Finally, we classify all the Artinian rings $R$ for which $\mathcal{E} \mathcal{G}(R)$ is isomorphic to some well-known graphs.
\end{abstract}

Keywords: Annihilating-ideal graph, zero-divisor graph, complete graph, planar graph, genus of a graph.

Math. Subj. Class. (2020): 13A15, 05C10, 05C12, 05C25

\section{Introduction}

Throughout this paper all rings are commutative rings (not a field) with unit element such that $1 \neq 0$. For a commutative ring $R$, we use $\mathbb{I}(R)$ to denote the set of ideals of $R$ and $\mathbb{I}^{*}(R)=\mathbb{I}(R) \backslash\{0\}$. An ideal $I$ of $R$ is said to be non-trivial if it is nonzero and proper both. An ideal $I$ of $R$ is said to be annihilator ideal if there is a nonzero ideal $J$ of $R$ such that $I J=0$. For $X \subseteq R$, we define annihilator of $X$ as $A n n(X)=\{r \in R: r X=0\}$. We use $A(R)$ to denote the set of annihilator ideas of $R$ and $A^{*}(R)=A(R) \backslash\{0\}$. We denote the set of zero-divisors, the set of nilpotent elements, the set of maximal ideals, the set of minimal prime ideals, and the set of Jacobson radical of a ring $R$ by $Z(R), \operatorname{Nil}(R)$,

\footnotetext{
*The authors are greatly indebted to the referee for his/her constructive comments and suggestion, which improves the quality of the paper a lot.

$\dagger$ Corresponding author.

E-mail addresses: mnazim1882@gmail.com (Mohd Nazim), nu.rehman.mm@amu.ac.in (Nadeem ur Rehman)
}

(c)(7) This work is licensed under https://creativecommons.org/licenses/by/4.0/ 
$\operatorname{Max}(R), \operatorname{Min}(R)$ and $J(R)$, respectively. A nonzero ideal $I$ of $R$ is called essential, denoted by $I \leq_{e} R$, if $I$ has a nonzero intersection with every nonzero ideal of $R$. Also, if $I$ is not an essential ideal of $R$ then, it is denoted by $I \not Z_{e} R$. A ring $R$ is said to be reduced, if it has no nonzero nilpotent element. For a nonzero nilpotent element $x$ of $R$, we use $\eta$ to denote the index of nilpotency of $x$. If $S$ is any subset of $R$, then $S^{*}$ denote the set $S \backslash\{0\}$. For any undefined notation or terminology in ring theory, we refer the reader to see [9].

Let $G$ be a graph with vertex set $V(G)$. The distance between two vertices $u$ and $v$ of $G$ denoted by $d(u, v)$, is the smallest path from $u$ to $v$. If there is no such path, then $d(u, v)=\infty$. The diameter of $G$ is defined as $\operatorname{diam}(G)=\sup \{d(u, v): u, v \in V(G)\}$. A cycle is a closed path in $G$. The girth of $G$ denoted by $\operatorname{gr}(G)$ is the length of a shortest cycle in $G(\operatorname{gr}(G)=\infty$ if $G$ contains no cycle). A graph is said to be complete if all its vertices are adjacent to each other. A complete graph with $n$ vertices is denoted by $K_{n}$. If $G$ is a graph such that the vertices of $G$ can be partitioned into two nonempty disjoint sets $U_{1}$ and $U_{2}$ such that vertices $u$ and $v$ are adjacent if and only if $u \in U_{1}$ and $v \in U_{2}$, then $G$ is called a complete bipartite graph. A complete bipartite graph with disjoint vertex sets of size $m$ and $n$, respectively, is denoted by $K_{m, n}$. We write $K_{n, \infty}$ (respectively, $K_{\infty, \infty}$ ) if one (respectively, both) of the disjoint vertex sets is infinite. A complete bipartite graph of the form $K_{1, n}$ is called a star graph. A graph $G$ is said to be planar if it can be drawn in the plane so that its edges intersect only at their ends. A subdivision of a graph is a graph obtained from it by replacing edges with pairwise internally-disjoint paths. A remarkably simple characterization of planar graphs was given by Kuratowski in 1930. Kuratowski's Theorem says that a graph $G$ is planar if and only if it contains no subdivision of $K_{5}$ or $K_{3,3}$. The genus of a graph $G$, denoted by $\gamma(G)$, is the minimum integer $k$ such that the graph can be drawn without crossing itself on a sphere with $k$ handles (i.e. an oriented surface of genus $k$ ). Thus, a planar graph has genus 0 , because it can be drawn on a sphere without self-crossing. For more details on graph theory, we refer to reader to see [21, 22].

The concept of zero-divisor graph of a commutative ring $R$, denoted by $\Gamma(R)$, was introduced by I. Beck [10]. The vertex set of $\Gamma(R)$ is $Z^{*}(R)=Z(R) \backslash\{0\}$ (set of nonzero zero-divisors of $R$ ) and two distinct vertices $x$ and $y$ are adjacent if and only if $x y=$ 0 , for details see [5, 8, 7]. In [14], Dol žan and Oblak also obtained several interesting results related with zero-divisor graph of rings and semirings. The zero-divisor graph of a noncommutative ring has been introduced and studied by Redmond [18], whereas the same concept for semigroup by Demeyer et al. [13].

In [11], Behboodi et al. generalized the zero-divisor graph to ideals by defining the annihilating-ideal graph $A G(R)$, with vertex set is $A^{*}(R)$ and two distinct vertices $I_{1}$ and $I_{2}$ are adjacent if and only if $I_{1} I_{2}=0$. For more details on annihilating-ideal graph, we refer the reader to see $[1,2,3,4,6,12,16]$.

In [17], M. Nikmehr et al. introduced the essential graph $E G(R)$ with vertex set $Z^{*}(R)=Z(R) \backslash\{0\}$ and two distinct vertices $x$ and $y$ are adjacent if and only if $a n n_{R}(x y)$ is an essential ideal of $R$.

Motivated by [17], we define the essential annihilating-ideal graph of $R$ denoted by $\mathcal{E} \mathcal{G}(R)$ with vertex set $A^{*}(R)$ and two distinct vertices $I_{1}$ and $I_{2}$ adjacent if and only if $\operatorname{Ann}\left(I_{1} I_{2}\right)$ is an essential ideal of $R$. In this paper we first prove that $A G(R)$ is a subgraph of $\mathcal{E} \mathcal{G}(R)$ and then studied some basic properties of $\mathcal{E} \mathcal{G}(R)$ such as connectedness, diameter, girth and shows that $\mathcal{E} \mathcal{G}(R)$ is a connected graph with $\operatorname{diam}(\mathcal{E} \mathcal{G}(R)) \leq 3$ and $\operatorname{gr}(\mathcal{E} \mathcal{G}(R)) \leq 4$, if $\mathcal{E} \mathcal{G}(R)$ contains a cycle. In the third section, we determine some condi- 
tions on $R$ under which $\mathcal{E} \mathcal{G}(R)$ is a star graph or a complete graph. In the last, we identify all the Artinian rings $R$ for which $\mathcal{E} \mathcal{G}(R)$ is isomorphic to some well-known graphs.

\section{Basic properties of essential annihilating-ideal graph}

We begin this section with the following lemma given by [17].

Lemma 2.1 ([17, Lemma 2.1]). Let $R$ be a commutative ring and I be an ideal of $R$. Then

(1) $I+\operatorname{Ann}(I)$ is an essential ideal of $R$.

(2) If $I^{2}=(0)$, then Ann $(I)$ is an essential ideal of $R$.

(3) If $R$ contains no proper essential ideals, then $J(R)=(0)$.

The following lemma is analogue of [17, Lemma 2.2].

Lemma 2.2. Let $R$ be a commutative ring. Then

(1) If $I_{1}$ and $I_{2}$ are adjacent in $A G(R)$, then $I_{1}$ and $I_{2}$ are also adjacent in $\mathcal{E} \mathcal{G}(R)$.

(2) If $I^{2}=0$ for some $I \in A^{*}(R)$, then $I$ is adjacent to every other vertex in $\mathcal{E} \mathcal{G}(R)$.

Proof. (1) Suppose $I_{1}$ and $I_{2}$ are adjacent in $A G(R)$, then $I_{1} I_{2}=0$ and so $\operatorname{Ann}\left(I_{1} I_{2}\right)=R$, is an essential ideal of $R$. Thus $I_{1}$ and $I_{2}$ are also adjacent in $\mathcal{E} \mathcal{G}(R)$.

(2) Suppose that $I^{2}=0$ for some $I \in A^{*}(R)$. Then by Lemma 2.1(2), Ann $(I)$ is an essential ideal of $R$. Since $A n n(I) \subseteq A n n(I J)$ for every $J \in A^{*}(R)$, therefore $A n n(I J)$ is also an essential ideal of $R$. Thus $I$ is adjacent to every other vertex of $\mathcal{E} \mathcal{G}(R)$.

Let $R$ be a commutative ring. By [11, Theorem 2.1], the annihilating ideal graph $A G(R)$ is a connected graph with $\operatorname{diam}(A G(R)) \leq 3$. Moreover, if $A G(R)$ contains a cycle, then $\operatorname{gr}(A G(R)) \leq 4$.

In view of part (1) of Lemma 2.2, we have the following result.

Theorem 2.3. Let $R$ be a commutative ring. Then $\mathcal{E} \mathcal{G}(R)$ is connected with diam $(\mathcal{E} \mathcal{G}(R)) \leq$ 3. Moreover, if $\mathcal{E} \mathcal{G}(R)$ contain a cycle, then $\operatorname{gr}(\mathcal{E} \mathcal{G}(R)) \leq 4$.

In Lemma 2.2(1), we proved that $A G(R)$ is a spanning subgraph of $\mathcal{E} \mathcal{G}(R)$ but this containment may be proper. The following examples shows that $A G(R)$ and $\mathcal{E} \mathcal{G}(R)$ are not identical.

\section{Example 2.4.}

1. If $R=\mathbb{Z}_{16}$, then $A G(R)$ is $P_{3}$ and $\mathcal{E} \mathcal{G}(R)$ is $K_{3}$.

2. If $R=\mathbb{Z}_{p^{5}}$, where $p$ is a prime number. Then $A G(R)$ is the following graph and $\mathcal{E} \mathcal{G}(R)$ is $K_{4}$.

Theorem 2.5. Let $R$ be a commutative reduced ring. Then $\mathcal{E} \mathcal{G}(R)=A G(R)$.

Proof. Clearly, $A G(R) \subseteq \mathcal{E} \mathcal{G}(R)$. We just have to prove that $\mathcal{E} \mathcal{G}(R)$ is a subgraph of $A G(R)$. Suppose on contrary that $I_{1} \sim I_{2}$ is an edge of $\mathcal{E} \mathcal{G}(R)$ such that $I_{1} I_{2} \neq 0$. Since $R$ is a reduced ring, then $I_{1} I_{2} \cap \operatorname{Ann}\left(I_{1} I_{2}\right)=0$, which implies that $\operatorname{Ann}\left(I_{1} I_{2}\right)$ is not an essential ideal of $R$, a contradiction. Thus $I_{1} I_{2}=0$ and $\mathcal{E} \mathcal{G}(R)=A G(R)$. 


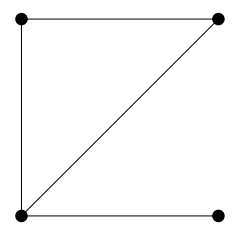

Figure 1: The graph $A G\left(\mathbb{Z}_{p^{5}}\right)$.

Theorem 2.6 ([12, Theorem 1.9(3)]). Let $R$ be a commutative ring with finitely many minimal primes. Then $\operatorname{diam}(A G(R))=2$ if and only if either $R$ is reduced with exactly two minimal primes and at least three nonzero annihilating-ideals, or $R$ is not reduced, $Z(R)$ is an ideal whose square is not $(0)$ and for each pair of annihilating-ideals $I_{1}$ and $I_{2}, I_{1}+I_{2}$ is an annihilating-ideal.

Theorem 2.7. Let $R$ be a commutative ring with $|\operatorname{Min}(R)|<\infty$. Then

(1) If $R$ is reduced ring, then $\operatorname{diam}(\mathcal{E} \mathcal{G}(R))=2$ if and only if $|\operatorname{Min}(R)|=2$ and $R$ has at least three nonzero annihilating-ideals. Moreover, in this case $\operatorname{gr}(\mathcal{E} \mathcal{G}(R)) \in\{4, \infty\}$.

(2) If $R$ is non-reduced, then $\operatorname{diam}(\mathcal{E} \mathcal{G}(R)) \leq 2$. Moreover, in this case $\operatorname{gr}(\mathcal{E} \mathcal{G}(R)) \in\{3, \infty\}$.

Proof. (1) First part is clear from Theorems 2.5 and 2.6. Now, let $\operatorname{Min}(R)=\left\{P_{1}, P_{2}\right\}$, then $\mathcal{E} \mathcal{G}(R)$ is a complete bipartite graph with partitions $V_{1}=\left\{I \in V(\mathcal{E} \mathcal{G}): I \subseteq P_{1}\right\}$ and $V_{2}=\left\{I \in V(\mathcal{E G}): I \subseteq P_{2}\right\}$ by [12, Theorem 1.2]. Hence $\operatorname{gr}(\mathcal{E} \mathcal{G}(R)) \in\{4, \infty\}$.

(2) Since $R$ is a non-reduced ring, then there is $I_{1} \in A^{*}(R)$ such that $I_{1}^{2}=0$. Thus by Lemma 2.2(2), $I_{1}$ is adjacent to every other vertex of $\mathcal{E} \mathcal{G}(R)$. Hence $\operatorname{diam}(\mathcal{E} \mathcal{G}(R)) \leq 2$. Also, if there are $I, J \in V(\mathcal{E} \mathcal{G}(R)) \backslash\left\{I_{1}\right\}$ such that $I \sim J$ is an edge of $\mathcal{E} \mathcal{G}(R)$, then $I_{1} \sim I \sim J \sim I_{1}$ is a triangle in $\mathcal{E} \mathcal{G}(R)$. Thus, $\operatorname{gr}(\mathcal{E} \mathcal{G}(R))=3$, otherwise $\operatorname{gr}(\mathcal{E} \mathcal{G}(R))=\infty$.

\section{Completeness of essential annihilating-ideal graph}

In this section, we characterize commutative rings $R$ for which $\mathcal{E} \mathcal{G}(R)$ is a star graph or a complete graph. We begin with the following lemma.

Lemma 3.1. Let $R$ be a commutative nonreduced ring. Then

(1) For every nilpotent ideal $I_{1}$ of $R, I_{1}$ is adjacent to every other vertex of $\mathcal{E} \mathcal{G}(R)$.

(2) The subgraph induced by the nilpotent ideals of $R$ is a complete subgraph of $\mathcal{E} \mathcal{G}(R)$.

Proof. (1) Suppose that $I_{1}$ be any nilpotent ideal of $R$. Let $I_{2} \in A^{*}(R)$. We show that $\operatorname{Ann}\left(I_{1} I_{2}\right) \leq_{e} R$. Since $\operatorname{Ann}\left(I_{1}\right) \subseteq \operatorname{Ann}\left(I_{1} I_{2}\right)$, then it is enough to show that $\operatorname{Ann}\left(I_{1}\right) \leq_{e} R$. Suppose on contrary that $\operatorname{Ann}\left(I_{1}\right) \mathbb{L}_{e} R$, then there exists $I_{3} \in \mathbb{I}^{*}(R)$ such that $\operatorname{Ann}\left(I_{1}\right) \cap I_{3}=0$, which implies that $r I_{1} \neq 0$ for every $r \in I_{3}^{*}$. Since $0 \neq r I_{1} \subseteq I_{3}$, then $I_{1} \cdot r I_{1}=r I_{1}^{2} \neq 0$. Continuing this process, we get $r I_{1}^{n} \neq 0$, for every positive integer $n$, which is a contradiction. This complete the proof.

(2) It is clear from (1). 
Lemma 3.2. Let $(R, \mathfrak{m})$ be a commutative Artinian local ring. Then $\mathcal{E} \mathcal{G}(R)$ is a complete graph.

Proof. Follows from Lemma 3.1.

Lemma 3.3. Let $R$ be a commutative decomposable ring. Then $\mathcal{E} \mathcal{G}(R)$ is a star graph if and only if $R=F \times D$, where $F$ is a field and $D$ is an integral domain.

Proof. $(\Rightarrow)$ Suppose that $\mathcal{E} \mathcal{G}(R)$ is a star graph and let $R=R_{1} \times R_{2}$, where $R_{1}$ and $R_{2}$ are commutative rings. If $R_{1}$ and $R_{2}$ both are not fields and $I_{1} \in \mathbb{I}^{*}\left(R_{1}\right), I_{2} \in \mathbb{I}^{*}\left(R_{2}\right)$, then $\left(R_{1} \times(0)\right) \sim\left((0) \times R_{2}\right) \sim\left(I_{1} \times(0)\right) \sim\left((0) \times I_{2}\right) \sim\left(R_{1} \times(0)\right)$ is a cycle of length 4 in $\mathcal{E} \mathcal{G}(R)$, a contradiction. Thus, without loss of generality we can assume that $R_{1}$ is a field. We claim that $R_{2}$ is an integral domain. Suppose on contrary that $R_{2}$ is not an integral domain, then there exists $I_{3}, I_{4} \in \mathbb{I}^{*}\left(R_{1}\right)$ such that $I_{3} I_{4}=0$. If $I_{3} \neq I_{4}$, then $\left(R_{1} \times(0)\right) \sim\left((0) \times I_{3}\right) \sim\left((0) \times I_{4}\right) \sim\left(R_{1} \times(0)\right)$ is a triangle in $\mathcal{E} \mathcal{G}(R)$, a contradiction. Also, if $I_{3}=I_{4}$, then by Lemma 3.1, $\left(R_{1} \times(0)\right) \sim\left((0) \times I_{3}\right) \sim\left((0) \times R_{2}\right) \sim\left(R_{1} \times(0)\right)$ is a triangle in $\mathcal{E} \mathcal{G}(R)$, again a contradiction. This complete the proof.

$(\Leftarrow)$ is clear.

Theorem 3.4. Let $R$ be an Artinian commutative ring with atleast two non-trivial ideals. Then $\mathcal{E} \mathcal{G}(R)$ is a star graph if and only if $\mathcal{E} \mathcal{G}(R) \cong K_{2}$.

Proof. $(\Rightarrow)$ Suppose $\mathcal{E} \mathcal{G}(R)$ is a star graph. If $R$ is a local ring, then from Lemma 3.2, $\mathcal{E} \mathcal{G}(R)$ is a complete graph. Since $\mathcal{E} \mathcal{G}(R)$ is a star graph, therefore $\mathcal{E} \mathcal{G}(R) \cong K_{2}$. If $R$ is non-local ring, then it is decomposable. Thus by Lemma 3.3, $R=F \times D$, where $F$ is a field and $D$ is an integral domain. Since $R$ is Artinian ring, then $D$ is Artinian and hence is a field. Thus $\mathcal{E} \mathcal{G}(R) \cong K_{2}$.

$(\Leftarrow)$ is evident.

Theorem 3.5. Let $R$ be a commutative ring with at least two non-trivial ideals. Then $\mathcal{E} \mathcal{G}(R)$ is a star graph if and only if one of the following holds:

(1) R has exactly two non-trivial ideals.

(2) $R=F \times D$, where $F$ is a field and $D$ is an integral domain which is not a field.

(3) $R$ has a minimal ideal $I_{1}$ such that $I_{1}$ is not an essential ideal of $R, I_{1}^{2}=0$ and for any nonzero annihilating ideal $I_{2}$ of $R, \operatorname{Ann}\left(I_{2}\right)=I_{1}$.

Proof. $(\Rightarrow)$ Suppose $\mathcal{E} \mathcal{G}(R)$ is a star graph. If $\left|A^{*}(R)\right|<\infty$, then from [11, Theorem 1.1], $R$ is an Artinian ring. Thus, by Theorem 3.4, $\mathcal{E} \mathcal{G}(R) \cong K_{2}$ and hence (1) hold.

Now, let $\left|A^{*}(R)\right|=\infty$ and $I_{1}$ is adjacent to every other vertex of $\mathcal{E} \mathcal{G}(R)$. We show that $I_{1}$ is minimal ideal of $R$. Suppose on contrary that there exists $I_{2} \in \mathbb{I}^{*}(R)$ such that $I_{2} \subset I_{1}$. Let $I_{3} \in A^{*}(R) \backslash\left\{I_{1}, I_{2}\right\}$, then $\operatorname{Ann}\left(I_{1} I_{3}\right) \leq_{e} R$. Since $I_{2} I_{3} \subseteq I_{1} I_{3}$, then $\operatorname{Ann}\left(I_{2} I_{3}\right)$ is also essential ideal of $R$. This implies that $I_{2}$ is also adjacent to every other vertex of $\mathcal{E} \mathcal{G}(R)$, a contradiction. Now, following two cases occur:

Case I: $I_{1}^{2} \neq 0$. Then $I_{1}^{2}=I_{1}$, thus by Brauer's Lemma [15, p. 172, Lemma 10.22], $R$ is decomposable. Since $\left|A^{*}(R)\right|=\infty$ and $\mathcal{E} \mathcal{G}(R)$ is a star graph. Then from Lemma 3.3, $R=F \times D$, where $F$ is a field and $D$ is an integral domain which is not a field. Hence (2) hold.

Case II: $I_{1}^{2}=0$. Let $I_{2} \in A^{*}(R) \backslash\left\{I_{1}\right\}$. Then $I_{2} \neq A n n\left(I_{2}\right)$, otherwise $I_{2}^{2}=0$ 
implies that $I_{2}$ is also adjacent to every other vertex of $\mathcal{E} \mathcal{G}(R)$, a contradiction. Now, since $I_{2} \sim \operatorname{Ann}\left(I_{2}\right)$, then $\operatorname{Ann}\left(I_{2}\right)=I_{1}$. If $I_{1}$ is an essential ideal of $R$, then $\operatorname{Ann}\left(I_{2}\right)$ is also an essential ideal of $R$. This shows that $I_{2}$ is also adjacent with every other vertex of $\mathcal{E} \mathcal{G}(R)$, which is a contradiction to our assumption that $\mathcal{E} \mathcal{G}(R)$ is a star graph because we are assuming that $I_{1}$ is adjacent with every other vertex of $\mathcal{E} \mathcal{G}(R)$ and $I_{1} \neq I_{2}$. Hence $I_{1}$ is not an essential ideal of $R$.

$(\Leftarrow)$ If $R$ has exactly two non-trivial ideals, then $R$ is Artinian ring with $\left|A^{*}(R)\right|=2$. Since $\mathcal{E} \mathcal{G}(R)$ is connected, therefore $\mathcal{E} \mathcal{G}(R) \cong K_{2}$. If $R=F \times D$, where $F$ is a field and $D$ is an integral domain which is not a field, then from Lemma $3.3, \mathcal{E} \mathcal{G}(R)$ is a star graph. Now, suppose that $R$ has a minimal ideal $I_{1}$ such that $I_{1}$ is not an essential ideal of $R$, $I_{1}^{2}=0$ and for any nonzero annihilating ideal $I_{2}$ of $R, \operatorname{Ann}\left(I_{2}\right)=I_{1}$. Let $I_{2}, I_{3} \in$ $A^{*}(R) \backslash\left\{I_{1}\right\}$ such that $I_{2} \sim I_{3}$ in $\mathcal{E} \mathcal{G}(R)$. This implies that $\operatorname{Ann}\left(I_{2} I_{3}\right) \leq_{e} R$ and $\operatorname{Ann}\left(I_{2}\right)=I_{1}=\operatorname{Ann}\left(I_{3}\right)$. Since $\operatorname{Ann}\left(I_{2}\right)=\operatorname{Ann}\left(I_{3}\right)$ is not an essential of $R$, there exists a nonzero ideal $I_{4}$ of $R$ such that $A n n\left(I_{2}\right) \cap I_{4}=A n n\left(I_{3}\right) \cap I_{4}=0$. This shows that $r I_{2} \neq 0$ and $r I_{3} \neq 0$ for every $r \in I_{4}^{*}$. On the other hand, since $A n n\left(I_{2} I_{3}\right) \leq_{e} R$, then $\operatorname{Ann}\left(I_{2} I_{3}\right) \cap I_{4} \neq 0$. That is there exists $s \in I_{4}^{*}$ such that $s I_{2} I_{3}=0$. Now, observe that $s I_{2} \subseteq I_{4}^{*}$ satisfies $s I_{2} \subseteq A n n\left(I_{3}\right)$, which implies that $A n n\left(I_{3}\right) \cap I_{4} \neq 0$, a contradiction. This complete the proof.

Theorem 3.6. Let $R$ be a commutative Artinian ring. Then $\mathcal{E} \mathcal{G}(R)$ is a complete graph if and only if one of the following holds:

(1) $R=F_{1} \times F_{2}$, where $F_{1}$ and $F_{2}$ are fields.

(2) $R$ is a local ring.

Proof. $(\Rightarrow)$ Suppose that $\mathcal{E} \mathcal{G}(R)$ is a complete graph. Since $R$ is Artinian, then $R \cong R_{1} \times R_{2} \times \cdots \times R_{n}$, where $R_{i}$ is Artinian local ring for each $1 \leq i \leq n$. The following cases occur:

Case I: $n \geq 3$. Then $R_{1} \times(0) \times \cdots \times(0)$ and $R_{1} \times(0) \times R_{3} \times \cdots \times(0)$ are nonzero annihilating ideals of $R$ such that $\left(R_{1} \times(0) \times \cdots \times(0)\right) \not\left(R_{1} \times(0) \times R_{3} \times \cdots \times(0)\right)$ in $\mathcal{E} \mathcal{G}(R)$, a contradiction.

Case II: $n=2$. We show that $R_{1}$ and $R_{2}$ are fields. Suppose on contrary that $R_{1}$ is not a field with non-trivial maximal ideal $\mathfrak{m}$. Then $\operatorname{Ann}\left(\left((0) \times R_{2}\right) \cdot\left(\mathfrak{m} \times R_{2}\right)\right)=\operatorname{Ann}((0) \times$ $\left.R_{2}\right)=R_{1} \times(0)$, which is not an essential ideal of $R$. Thus $\left((0) \times R_{2}\right) \nsim\left(\mathfrak{m} \times R_{2}\right)$ in $\mathcal{E} \mathcal{G}(R)$, a contradiction. Hence (2) holds.

Case III: $n=1$. Then $R$ is Artinian local ring and (1) holds.

$(\Leftarrow)$ If $R$ is local, then from Lemma 3.2, $\mathcal{E} \mathcal{G}(R)$ is a complete graph. If $R=F_{1} \times F_{2}$, where $F_{1}$ and $F_{2}$ are fields, then $\mathcal{E} \mathcal{G}(R) \cong K_{2}$.

Theorem 3.7. Let $R$ be a commutative ring with at least one minimal ideal. Then $\mathcal{E} \mathcal{G}(R) \cong$ $K_{m, n}$, where $m, n \geq 2$ if and only if $R=D \times S$, where $D$ and $S$ are integral domains which are not fields.

Proof. $(\Rightarrow)$ Suppose that $\mathcal{E} \mathcal{G}(R) \cong K_{m, n}$, where $m, n \geq 2$. Let $I_{1}$ be minimal ideal of $R$. If $I_{1}^{2}=0$, then from Lemma $2.2, I_{1}$ is adjacent to every other vertex, a contradiction. Thus $I_{1}^{2} \neq 0$. Since $I_{1}$ is minimal, therefore $I_{1}^{2}=I_{1}$. Therefore, Brauer's Lemma [15, p. 172, Lemma 10.22], $R=R_{1} \times R_{2}$, where $R_{1}$ and $R_{2}$ are commutative rings. Now, our objective is to show that $R_{1}$ and $R_{2}$ are integral domains. Suppose on contrary that 
$R_{1}$ is not an integral domain with nonzero annihilating ideal $I_{2}$. As above, $I_{2}^{2} \neq 0$ which implies that $I_{2} \notin \operatorname{Ann}\left(I_{2}\right)$. Thus $\left(I_{2} \times(0)\right) \sim\left((0) \times R_{2}\right) \sim\left(\operatorname{Ann}\left(I_{2}\right) \times(0)\right) \sim\left(I_{2} \times(0)\right)$ is a triangle in $\mathcal{E} \mathcal{G}(R)$, a contradiction. Hence $R_{1}$ is an integral domain. Similarly, one can prove that $R_{2}$ is an integral domain. Since $m, n \geq 2$, therefore $R_{1}$ and $R_{2}$ are not fields. $(\Leftarrow)$ Suppose that $R=D \times S$, where $D$ and $S$ are integral domains which are not fields. Let $U=\left\{I_{1} \times(0): I_{1} \in \mathbb{I}^{*}(D)\right\}$ and $V=\left\{(0) \times I_{2}: I_{2} \in \mathbb{I}^{*}(S)\right\}$. Then $A^{*}(R)=U \cup V$ such that no two vertices of $U$ or $V$ are adjacent in $\mathcal{E G}(R)$. Also, every vertex of $U$ is adjacent to every vertex of $V$ in $\mathcal{E} \mathcal{G}(R)$. Thus, $\mathcal{E} \mathcal{G}(R) \cong K_{m, n}$. Since $D$ and $S$ are not fields, therefore $m, n \geq 2$.

Lemma 3.8. Let $R$ be a commutative ring. Then

(1) Let $I_{1}, I_{2}, I_{3} \in A^{*}(R)$ such that Ann $\left(I_{1}\right)=$ Ann $\left(I_{2}\right)$. Then $I_{1} \sim I_{3}$ is an edge of $\mathcal{E} \mathcal{G}(R)$ if and only if $I_{2} \sim I_{3}$ is an edge of $\mathcal{E} \mathcal{G}(R)$.

(2) Let $I \in A^{*}(R)$. Then Ann $(I) \leq_{e} R$ if and only if $A n n\left(I^{n}\right) \leq_{e} R$ for every $n \geq 2$. In particular, if $\operatorname{Ann}\left(I^{3}\right) \leq_{e} R$, then $A n n\left(I^{n}\right) \leq_{e} R$ for every $n \geq 1$.

Proof. (1) $(\Rightarrow)$ Suppose that $I_{1} \sim I_{3}$ is an edge of $\mathcal{E} \mathcal{G}(R)$, then $A n n\left(I_{1} I_{3}\right) \leq_{e} R$. We have to show that $\operatorname{Ann}\left(I_{2} I_{3}\right) \leq_{e} R$. Suppose on contrary that $A n n\left(I_{2} I_{3}\right)$ is not an essential ideal of $R$, then there exits $I_{4} \in \mathbb{I}^{*}(R)$ such that $\operatorname{Ann}\left(I_{2} I_{3}\right) \cap I_{4}=0$. This implies that $r I_{2} I_{3} \neq 0$ for all $r \in I_{4}^{*}$. On the other hand, since $\operatorname{Ann}\left(I_{1} I_{3}\right)$ is an essential ideal of $R$, then $\operatorname{Ann}\left(I_{1} I_{3}\right) \cap I_{4} \neq 0$. That is there exists some $s \in I_{4}^{*}$ such that $s I_{1} I_{3}=0$. Now, observe that $s I_{3} \subseteq I_{4}^{*}$ satisfies $s I_{3} \subseteq \operatorname{Ann}\left(I_{1}\right)=\operatorname{Ann}\left(I_{2}\right)$, which implies that $s I_{2} I_{3}=0$, a contradiction.

$(\Leftarrow)$ Using similar argument as above we get the required result.

$(2)(\Rightarrow)$ is clear.

$(\Leftarrow)$ Suppose on contrary that $\operatorname{Ann}(I)$ is not an essential ideal of $R$, then there exists nonzero ideal $I_{1}$ of $R$ such that $A n n(I) \cap I_{1}=0$. This implies that $r I \neq 0$ for all $r \in I_{1}^{*}$. On the other hand, since $\operatorname{Ann}\left(I^{2}\right) \leq_{e} R$, then $\operatorname{Ann}\left(I^{2}\right) \cap I_{1} \neq 0$. That is there exists some $s \in I_{1}^{*}$ such that $s I^{2}=0$. Now, observe that $r=s I \subseteq I_{1}^{*}$ such that $r I=0$, a contradiction.

For the particular case, we need to show that $\operatorname{Ann}\left(I^{2}\right) \leq_{e} R$. Suppose on contrary that there is some $I_{1} \in \mathbb{I}^{*}(R)$ such that $\operatorname{Ann}\left(I^{2}\right) \cap I_{1}=0$, which implies that $r I^{2} \neq 0$ for all $r \in I_{1}^{*}$. On the other hand, since $\operatorname{Ann}\left(I^{3}\right) \leq_{e} R$, then $\operatorname{Ann}\left(I^{3}\right) \cap I_{1} \neq 0$. That is there exists some $s \in I_{1}^{*}$ such that $s I^{3}=0$. Now, observe that $r=s I^{2} \subseteq I_{1}^{*}$ such that $r I=0$, which implies that $A n n(I) \cap I_{1} \neq 0$. Since $A n n(I)$ is a subset of $\operatorname{Ann}\left(I^{2}\right)$, then $\operatorname{Ann}\left(I^{2}\right) \cap I_{1} \neq 0$, a contradiction.

Theorem 3.9. Let $R$ be a commutative non-reduced ring. Then $\mathcal{E} \mathcal{G}(R)$ is a complete graph if and only if $A n n(I) \leq_{e} R$ for every $I \in A^{*}(R)$.

Proof. $(\Rightarrow)$ Suppose that $\mathcal{E} \mathcal{G}(R)$ is a complete graph. We claim that $R$ is indecomposable ring. Suppose on contrary that $R=R_{1} \times R_{2}$, where $R_{1}$ and $R_{2}$ are commutative rings. Since $R$ is non-reduced ring, without loss of generality, we can assume that $R_{1}$ is non-reduced ring with nonzero nilpotent element $x$. Let $I_{1}=x R_{1}$. Then $\operatorname{Ann}\left(\left(I_{1} \times R_{2}\right) \cdot\left((0) \times R_{2}\right)\right)=\operatorname{Ann}\left((0) \times R_{2}\right)=R_{1} \times(0)$, is not an essential ideal of $R$, a contradiction to the completeness of $\mathcal{E} \mathcal{G}(R)$. Let $I \in A^{*}(R)$ be arbitrary. If $I$ is nilpotent ideal, then from Lemma 3.1(1), $\operatorname{Ann}(I) \leq_{e} R$. Suppose $I$ is not nilpotent ideal. 
Since $R$ is indecomposable, then $I^{2} \neq I$, which implies that $\operatorname{Ann}\left(I^{3}\right) \leq_{e} R$. Hence by Lemma 3.8(2), $\operatorname{Ann}(I) \leq_{e} R$.

$(\Leftarrow)$ is evident.

\section{Essential annihilating-ideal graph as some special type of graphs}

In this section, we characterize all the Artinian rings $R$ for which $\mathcal{E} \mathcal{G}(R)$ is a tree, a unicycle graph, a split graph, a outerplanar graph, a planar graph and a toroidal graph.

Theorem 4.1. Let $R$ be a commutative Artinian ring (not a field). Then $\mathcal{E} \mathcal{G}(R)$ is a tree if and only if either $R \cong F_{1} \times F_{2}$, where $F_{1}$ and $F_{2}$ are fields or $R$ is a local ring with at most two non-trivial ideals.

Proof. Suppose that $\mathcal{E} \mathcal{G}(R)$ is a tree. Since $R$ is an Artinain ring, then $R \cong R_{1} \times R_{2} \times \cdots \times R_{n}$, where each $R_{i}$ is an Artinian local ring. If $n \geq 3$. Consider $I_{1}=$ $R_{1} \times(0) \times \cdots \times(0), I_{2}=(0) \times R_{2} \times(0) \times \cdots \times(0)$ and $I_{3}=(0) \times(0) \times R_{3} \times(0) \times \cdots \times(0)$. Then $I_{1} \sim I_{2} \sim I_{3} \sim I_{1}$ is a cycle of in $\mathcal{E} \mathcal{G}(\mathcal{R})$, a contradiction.

Suppose $n=2$, then we show that $R_{1}$ and $R_{2}$ both are fields. Suppose on contrary that $R_{1}$ is not a field with nonzero maximal ideal $\mathfrak{m}$. Consider $J_{1}=(0) \times R_{2}, J_{2}=\mathfrak{m} \times(0)$, $J_{3}=\mathfrak{m} \times R_{2}$ and $J_{4}=R_{1} \times(0)$. Then $J_{1} \sim J_{2} \sim J_{3} \sim J_{4} \sim J_{1}$ is a cycle in $\mathcal{E} \mathcal{G}(R)$, a contradiction.

If $n=1$, then $R$ is Artinian local ring. Thus by Lemma 3.2, $\mathcal{E} \mathcal{G}(R)$ is a complete graph. Since $\mathcal{E} \mathcal{G}(R)$ is a tree, therefore $R$ has at most two non-trivial ideal.

Converse is clear.

Theorem 4.2. Let $R$ be a commutative Artinian ring (not a field). Then $\mathcal{E} \mathcal{G}(R)$ is unicycle if and only if either $R \cong F_{1} \times F_{2} \times F_{3}$, where $F_{i}$ is a field for each $1 \leq i \leq 3$ or $R$ is an Artinain local ring with exactly three non-trivial ideals.

Proof. Suppose that $\mathcal{E} \mathcal{G}(R)$ is unicycle. Since $R$ is Artinian ring, then $R \cong R_{1} \times R_{2} \times \cdots \times R_{n}$, where $R_{i}$ is Artinian local ring for each $1 \leq i \leq n$. Let $n \geq 4$. Consider $I_{1}=R_{1} \times(0) \times \cdots \times(0), I_{2}=(0) \times R_{2} \times(0) \times \cdots \times(0)$, $I_{3}=(0) \times(0) \times R_{3} \times(0) \times \cdots \times(0)$ and $J_{1}=(0) \times(0) \times R_{3} \times(0) \times \cdots \times(0)$, $J_{2}=R_{1} \times R_{2} \times(0) \times \cdots \times(0), J_{3}=(0) \times(0) \times(0) \times R_{4} \times(0) \times \cdots \times(0)$. Then $I_{1} \sim I_{2} \sim I_{3} \sim I_{1}$ as well as $J_{1} \sim J_{2} \sim J_{3} \sim J_{1}$ are two different cycles in $\mathcal{E} \mathcal{G}(R)$, a contradiction. Hence $n \leq 3$.

First, let $n=3$ and suppose on contrary that $R_{2}$ is not a field with nonzero maximal ideal $\mathfrak{m}$. Consider $I_{1}=R_{1} \times(0) \times(0), I_{2}=(0) \times R_{2} \times(0), I_{3}=(0) \times(0) \times R_{3}$ and $J_{1}=R_{1} \times(0) \times(0), J_{2}=(0) \times \mathfrak{m} \times(0), J_{3}=(0) \times(0) \times R_{3}$. Then $I_{1} \sim I_{2} \sim I_{3} \sim I_{1}$ and $J_{1} \sim J_{2} \sim J_{3} \sim J_{1}$ are two different cycles in $\mathcal{E} \mathcal{G}(R)$, a contradiction. Hence $R_{i}$ is a field for each $1 \leq i \leq 3$.

Now, let $n=2$. If $R_{1}$ and $R_{2}$ both are fields then $\mathcal{E G}(R) \cong K_{2}$, a contradiction. Thus one of $R_{i}$ say $R_{2}$ is not a field with nonzero maximal ideal $\mathfrak{m}$. Then $\left(R_{1} \times(0)\right) \sim((0) \times \mathfrak{m}) \sim$ $\left((0) \times R_{2}\right) \sim\left(R_{1} \times(0)\right)$ as well as $\left(R_{1} \times \mathfrak{m} \sim((0) \times \mathfrak{m}) \sim\left((0) \times R_{2}\right) \sim\left(R_{1} \times \mathfrak{m}\right)\right.$ are two different cycles in $\mathcal{E} \mathcal{G}(R)$, again a contradiction.

If $n=1$, then $R$ is an Artinian local ring. Thus, by Lemma 3.2, $\mathcal{E G}(R)$ is a complete graph. Since $\mathcal{E} \mathcal{G}(R)$ is unicycle, $R$ have exactly three non-trivial ideals.

Theorem 4.3 ([21]). Let $G$ be a connected graph. Then $G$ is a split graph if and only if $G$ contains no induced subgraph isomorphic to $2 K_{2}, C_{4}, C_{5}$. 
Theorem 4.4. Let $R$ be a commutative Artinian non-local ring. Then $\mathcal{E} \mathcal{G}(R)$ is split graph if and only if either $R \cong F_{1} \times F_{2} \times F_{3}$ or $R \cong F_{1} \times F_{2}$, where $F_{i}$ is a field for each $1 \leq i \leq 3$.

Proof. Suppose that $\mathcal{E} \mathcal{G}(R)$ is a split graph. Since $R$ is Artinian non-local ring, then $R \cong R_{1} \times R_{2} \times \cdots \times R_{n}$, where each $R_{i}$ is an Artinian local ring and $n \geq 2$. If $n \geq 4$, then $I_{1}=R_{1} \times R_{2} \times(0) \times \cdots \times(0) \sim J_{1}=(0) \times(0) \times R_{3} \times R_{4} \times(0) \times \cdots \times(0)$ and $I_{2}=R_{1} \times(0) \times R_{3} \times(0) \times \cdots \times(0) \sim J_{2}=(0) \times R_{2} \times(0) \times R_{4} \times(0) \times \cdots \times(0)$ induces $2 K_{2}$ in $\mathcal{E} \mathcal{G}(R)$, a contradiction. Hence $n=2$ or 3 . We have following cases:

Case I: If $n=3$, then we show that each $R_{i}$ ia a field. Suppose on contrary that $R_{1}$ is not a field with nonzero maximal ideal $\mathfrak{m}$. Then $\left(R_{1} \times(0) \times(0)\right) \sim\left((0) \times R_{2} \times R_{3}\right) \sim$ $(\mathfrak{m} \times(0) \times(0)) \sim\left((0) \times R_{2} \times(0)\right) \sim\left(R_{1} \times(0) \times(0)\right)$ is $C_{4}$ in $\mathcal{E} \mathcal{G}(R)$, a contradiction. Hence $R_{i}$ is a field for each $1 \leq i \leq 3$.

Case II: Let $n=2$ and suppose that $R_{2}$ is not a field with nonzero maximal ideal $\mathfrak{m}^{\prime}$. Then $\left(R_{1} \times(0)\right) \sim\left((0) \times R_{2}\right) \sim\left(R_{1} \times \mathfrak{m}^{\prime}\right) \sim\left((0) \times \mathfrak{m}^{\prime}\right) \sim\left(R_{1} \times(0)\right)$ is $C_{4}$ in $\mathcal{E} \mathcal{G}(R)$, a contradiction. Hence $R_{1}$ and $R_{2}$ both are fields.

Converse is clear.

Theorem 4.5 ([22]). A graph $G$ is outerplanar if and only if it does not contain a subdivision of $K_{4}$ or $K_{2,3}$.

Theorem 4.6. Let $R$ be a commutative Artinian ring. Then $\mathcal{E} \mathcal{G}(R)$ is outerplanar if and only if one of the following holds:

(1) $R=F_{1} \times F_{2} \times F_{3}$, where $F_{i}$ is a field for each $1 \leq i \leq 3$.

(2) $R=F_{1} \times F_{2}$, where $F_{1}$ and $F_{2}$ are fields.

(3) $R=F \times R_{1}$, where $F$ is a field and $\left(R_{1}, \mathfrak{m}\right)$ is a local ring with $\mathfrak{m}$ is the only non-trivial ideal of $R_{1}$.

(4) $R$ is a local ring with at most three non-trivial ideals.

Proof. Suppose that $\mathcal{E} \mathcal{G}(R)$ is outerplanar. Since $R$ is Artinian ring, then $R \cong R_{1} \times R_{2} \times \cdots \times R_{n}$, where each $R_{i}$ is Artinian local ring. If $n \geq 4$, then the set $\left\{I_{1}=\right.$ $R_{1} \times(0) \times \cdots \times(0), I_{2}=(0) \times R_{2} \times(0) \times \cdots \times(0), I_{3}=(0) \times(0) \times R_{3} \times(0) \times \cdots \times(0)$, $\left.I_{4}=(0) \times(0) \times(0) \times R_{4} \times(0) \times \cdots \times(0)\right\}$ induces $K_{4}$ in $\mathcal{E} \mathcal{G}(R)$, a contradiction. Hence $n \leq 3$. The following cases occur:

Case I: $n=3$. We claim that $R_{i}$ is a field for each $1 \leq i \leq 3$. Suppose on contrary that $R_{2}$ is not a field with nonzero maximal ideal $\mathfrak{m}$. Then the set $\left\{R_{1} \times(0) \times(0), R_{1} \times \mathfrak{m} \times(0),(0) \times \mathfrak{m} \times(0),(0) \times(0) \times R_{3},(0) \times R_{2} \times R_{3}\right\}$ induces a copy of $K_{2,3}$ with partition sets $A=\left\{(0) \times(0) \times R_{3},(0) \times R_{2} \times R_{3}\right\}$ and $B=\left\{R_{1} \times(0) \times(0), R_{1} \times \mathfrak{m} \times(0),(0) \times \mathfrak{m} \times(0)\right\}$, a contradiction. Therefore $R_{i}$ is a field for each $1 \leq i \leq 3$.

Case II: $n=2$ and let $R_{i}$ is not a field with nonzero maximal ideal $\mathfrak{m}_{i}$ for each $i=$ 1,2 . Then the set $\left\{R_{1} \times(0),(0) \times R_{2}, \mathfrak{m}_{1} \times(0),(0) \times \mathfrak{m}_{2}\right\}$ induces a copy of $K_{4}$ in $\mathcal{E} \mathcal{G}(R)$, a contradiction. Hence one of $R_{i}$ (say $R_{1}$ ) must be a field. Let $I$ be a non-trivial ideal of $R_{2}$ other than maximal ideal $\mathfrak{m}_{2}$. Then the set $\left\{R_{1} \times(0), R_{1} \times \mathfrak{m}_{2},(0) \times R_{2}\right.$, $\left.(0) \times \mathfrak{m}_{2},(0) \times I\right\}$ induces a copy of $K_{2,3}$ with partition sets $A=\left\{R_{1} \times(0), R_{1} \times \mathfrak{m}_{2}\right\}$ and $B=\left\{(0) \times R_{2},(0) \times \mathfrak{m}_{2},(0) \times I\right\}$ in $\mathcal{E} \mathcal{G}(R)$, a contradiction. Hence $R_{2}$ is a field or 
has unique non-trivial ideal.

Case III: $n=1$, then $R$ is an Artinian local ring. Thus by Lemma 3.2, $\mathcal{E G}(R)$ is a complete graph. Since $\mathcal{E} \mathcal{G}(R)$ is outerplanar, $R$ have at most three non-trivial ideals.

Converse follows from Lemma 3.2, Theorem 4.5, Figures 2 and 3.

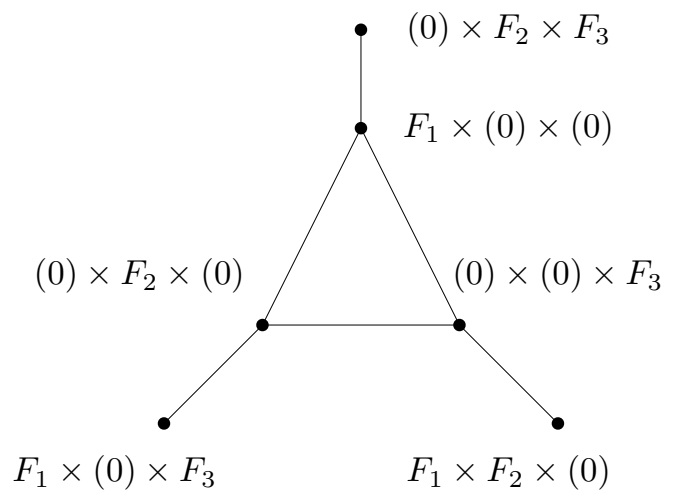

Figure 2: The graph $\mathcal{E} \mathcal{G}\left(F_{1} \times F_{2} \times F_{3}\right)$.

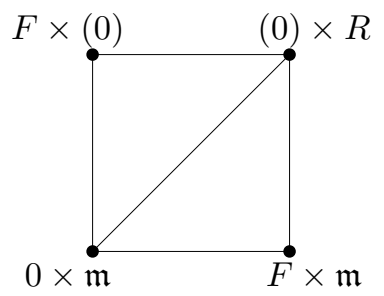

Figure 3: The graph $\mathcal{E} \mathcal{G}\left(F \times R_{1}\right)$, where $\mathfrak{m}$ is the only non-trivial ideal of $R_{1}$.

Lemma 4.7 ([20, Proposition 2.7]). If $(R, \mathfrak{m})$ is an Artinian local ring and there is an ideal $I$ of $R$ such that $I \neq \mathfrak{m}^{i}$ for every $i$, then $R$ has at least three distinct non-trivial ideals $J, K$ and $L$ such that $J, K, L \neq \mathfrak{m}^{i}$ for each $i$.

Theorem 4.8 (Kuratowski's Theorem). A graph $G$ is planar if and only if it contains no subdivision of $K_{5}$ or $K_{3,3}$.

Lemma 4.9. Let $(R, \mathfrak{m})$ be a commutative Artinian local ring. Then $\mathcal{E} \mathcal{G}(R)$ is planar if and only if $R$ have at most four non-trivial ideals.

Proof. It is clear from Lemma 3.2 and Theorem 4.8.

Theorem 4.10. Let $R$ be a commutative Artinian ring. Then $\mathcal{E} \mathcal{G}(R)$ is planar graph if and only if one of the following hold:

(1) $R=F_{1} \times F_{2} \times F_{3}$, where $F_{i}$ is a field for each $1 \leq i \leq 3$. 
(2) R has at most four non-trivial ideals.

Proof. Suppose that $\mathcal{E} \mathcal{G}(R)$ is a planar graph. If $\left|A^{*}(R)\right| \leq 4$, then (2) holds. Thus, we assume that $\left|A^{*}(R)\right| \geq 5$. Since $R$ is Artinian ring, then $R \cong R_{1} \times R_{2} \times \cdots \times R_{n}$, where each $R_{i}$ is Artinian local ring. If $n \geq 4$, then the set $\left\{R_{1} \times(0) \times \cdots \times(0), R_{1} \times R_{2} \times\right.$ $\left.(0) \times \cdots \times(0),(0) \times R_{2} \times(0) \times \cdots \times(0)\right\} \cup\left\{(0) \times(0) \times R_{3} \times R_{4} \times(0) \times \cdots \times(0),(0) \times\right.$ $\left.(0) \times R_{3} \times(0) \times \cdots \times(0),(0) \times(0) \times(0) \times R_{4} \times(0) \times \cdots \times(0)\right\}$ induces a copy of $K_{3,3}$ in $\mathcal{E} \mathcal{G}(R)$, a contradiction. Hence $n \leq 3$. The following cases occur:

Case I: $n=3$. We claim that $R_{i}$ is a field for each $1 \leq i \leq 3$. Suppose on contrary that one of $R_{i}$ say $R_{2}$ is not a field with nonzero maximal ideal $\mathfrak{m}$. Then the set $\left\{R_{1} \times(0) \times(0), R_{1} \times \mathfrak{m} \times(0),(0) \times \mathfrak{m} \times(0),(0) \times \mathfrak{m} \times R_{3},(0) \times(0) \times R_{3},(0) \times R_{2} \times R_{3}\right\}$ induces a copy of $K_{3,3}$ with partition sets $A=\left\{R_{1} \times(0) \times(0), R_{1} \times \mathfrak{m} \times(0),(0) \times \mathfrak{m} \times(0)\right\}$ and $B=\left\{(0) \times(0) \times R_{3},(0) \times \mathfrak{m} \times R_{3},(0) \times R_{2} \times R_{3}\right\}$ in $\mathcal{E} \mathcal{G}(R)$, a contradiction. Hence, (1) satisfied.

Case II: $n=2$. Since $\left|A^{*}(R)\right| \geq 5$, then one of $R_{i}$ is not a field for some $i=1,2$. Suppose that $R_{1}$ is not a field with nonzero maximal ideal $\mathfrak{m}_{1}$. If $R_{2}$ is a field, then $\left|A^{*}(R)\right| \geq 5$ shows that $R_{1}$ have at least two non-trivial ideals. Let $I$ be a non-trivial ideal of $R_{1}$ other than the maximal ideal. Then the set $\left\{R_{1} \times(0), \mathfrak{m}_{1} \times(0), I \times(0)\right\} \cup\left\{(0) \times R_{2}\right.$, $\left.\mathfrak{m}_{1} \times R_{2}, I \times R_{2}\right\}$ induces a copy of $K_{3,3}$ in $\mathcal{E} \mathcal{G}(R)$, a contradiction.

Now, if $R_{2}$ is not a field with nonzero maximal ideal $\mathfrak{m}_{2}$, then the set $\left\{R_{1} \times(0),(0) \times \mathfrak{m}_{2}, R_{1} \times \mathfrak{m}_{2}\right\} \cup\left\{(0) \times R_{2}, \mathfrak{m}_{1} \times(0), \mathfrak{m}_{1} \times R_{2}\right\}$ induces a copy of $K_{3,3}$ in $\mathcal{E} \mathcal{G}(R)$, again a contradiction.

Case III: $n=1$. Then $R$ is an Artinian local ring. Thus, by Lemma 3.2, $\mathcal{E} \mathcal{G}(R)$ is a complete graph. Since $\left|A^{*}(R)\right| \geq 5$, then $\mathcal{E} \mathcal{G}(R)$ contains a copy of $K_{5}$, which is a contradiction.

Conversely, If $R$ is an Artinian ring with at most four non-trivial ideals, then by Theorem 4.8, $\mathcal{E} \mathcal{G}(R)$ is planar. Also, if $R=F_{1} \times F_{2} \times F_{3}$, where $F_{i}$ is a field for each $1 \leq i \leq 3$, then from Figure $2, \mathcal{E} \mathcal{G}(R)$ is planar.

Lemma 4.11 ([22]). $\gamma\left(K_{n}\right)=\left\lceil\frac{1}{12}(n-3)(n-4)\right\rceil$, where $\lceil x\rceil$ is the least integer that is greater than or equal to $x$. In particular, $\gamma\left(K_{n}\right)=1$ if $n=5,6,7$.

Lemma 4.12 ([22]). $\gamma\left(K_{m, n}\right)=\left\lceil\frac{1}{4}(m-2)(n-2)\right\rceil$, where $\lceil x\rceil$ is the least integer that is greater than or equal to $x$. In particular, $\gamma\left(K_{4,4}\right)=\gamma\left(K_{3, n}\right)=1$ if $n=3,4,5,6$.

Theorem 4.13. Let $(R, \mathfrak{m})$ be a commutative Artinian local ring. Then $\gamma(\mathcal{E} \mathcal{G}(R))=1$ if and only if $R$ have at least five and at most seven non-trivial ideals.

Proof. Since $(R, \mathfrak{m})$ is an Artinian local ring, then from Lemma 3.2, $\mathcal{E} \mathcal{G}(R)$ is a complete graph. Thus, by Lemma 4.11, $5 \leq r \leq 7$, where $r$ is the number of non-trivial ideals of $R$.

Theorem 4.14. Let $R$ be a commutative Artinian ring such that $R=F_{1} \times F_{2} \times \cdots \times F_{n}$, where $n \geq 4$ and $F_{i}$ is a field for each $1 \leq i \leq n$. Then $\gamma(\mathcal{E G}(R))=1$ if and only if $n=4$.

Proof. Since $R$ is a reduced $\operatorname{ring}, \mathcal{E} \mathcal{G}(R)=A G(R)$ by Theorem 2.5. Hence the result follows from [19, Theorem 2]. 
Theorem 4.15. Let $R$ be a commutative Artinian ring such that $R=R_{1} \times R_{2} \times \cdots \times R_{n}$, where $n \geq 2$ and each $\left(R_{i}, \mathfrak{m}_{i}\right)$ is an Artinian local ring with $m_{i} \neq 0$. Let $\eta_{i}$ be the nilpotency of $\mathfrak{m}_{i}$. Then $\gamma(\mathcal{E G}(R))=1$ if and only if $n=2$ and $\mathfrak{m}_{1}$ and $\mathfrak{m}_{2}$ are the only non-trivial ideals of $R_{1}$ and $R_{2}$ respectively.

Proof. Suppose that $\gamma(\mathcal{E} \mathcal{G}(R))=1$. If $n \geq 3$, then the set $\left\{\mathfrak{m}_{1}^{\eta_{1}-1} \times(0) \times \cdots \times(0),(0) \times\right.$ $\left.\mathfrak{m}_{2}^{\eta_{2}-1} \times(0) \times \cdots \times(0), \mathfrak{m}_{1}^{\eta_{1}-1} \times \mathfrak{m}_{2}^{\eta_{2}-1} \times(0) \times \cdots \times(0)\right\} \cup\left\{(0) \times(0) \times R_{3} \times(0) \times \cdots \times\right.$ $(0),(0) \times(0) \times \mathfrak{m}_{3} \times(0) \times \cdots \times(0), \mathfrak{m}_{1} \times \mathfrak{m}_{2} \times \mathfrak{m}_{3} \times(0) \times \cdots \times(0), \mathfrak{m}_{1} \times(0) \times \mathfrak{m}_{3} \times$ $(0) \times \cdots \times(0),(0) \times \mathfrak{m}_{2} \times \mathfrak{m}_{3} \times(0) \times \cdots \times(0), \mathfrak{m}_{1} \times(0) \times R_{3} \times(0) \times \cdots \times(0),(0) \times$ $\left.\mathfrak{m}_{2} \times R_{3} \times(0) \times \cdots \times(0)\right\}$ induces a copy of $K_{3,7}$ in $\mathcal{E} \mathcal{G}(R)$. Thus, from Lemma 4.12, $\gamma(\mathcal{E} \mathcal{G}(R))>1$, a contradiction. Hence $n=2$.

Suppose $I$ is non-trivial ideal of $R_{1}$ such that $I \neq \mathfrak{m}_{1}$. Then the set $\left\{R_{1} \times(0)\right.$, $\left.\mathfrak{m}_{1} \times(0), R_{1} \times \mathfrak{m}_{2}, I \times(0), I \times \mathfrak{m}_{2}\right\} \cup\left\{(0) \times R_{2},(0) \times \mathfrak{m}_{2}, \mathfrak{m}_{1} \times R_{2}, \mathfrak{m}_{1} \times \mathfrak{m}_{2}\right\}$ induces a copy of $K_{4,5}$ in $\mathcal{E} \mathcal{G}(R)$. By Lemma 4.12, $\gamma(\mathcal{E} \mathcal{G}(R))>1$, a contradiction. Hence $R_{1}$ has unique non-trivial ideal $\mathfrak{m}_{1}$. Similarly, we can show that $R_{2}$ has unique non-trivial ideal $\mathfrak{m}_{2}$.

Conversely, let $R=R_{1} \times R_{2}$, where $\mathfrak{m}_{1}$ and $\mathfrak{m}_{2}$ are the only non-trivial ideals of $R_{1}$ and $R_{2}$ respectively, then $\left|A^{*}(R)\right|=7$. It is easy to see that the set $\left\{R_{1} \times(0)\right.$, $\left.\mathfrak{m}_{1} \times(0), R_{1} \times \mathfrak{m}_{2}\right\} \cup\left\{(0) \times R_{2},(0) \times \mathfrak{m}_{2}, \mathfrak{m}_{1} \times R_{2}\right\}$ induces a copy of $K_{3,3}$, which implies that $K_{3,3} \leq \mathcal{E} \mathcal{G}(R) \leq K_{7}$. Hence, by Lemma 4.11 and 4.12, $\gamma(\mathcal{E} \mathcal{G}(R))=1$.

Theorem 4.16 ([19, Theorem 4]). Let $R=R_{1} \times R_{2} \times F$ be a commutative ring, where each $\left(R_{i}, \mathfrak{m}_{i}\right)$ is a local ring with $\mathfrak{m}_{i} \neq 0$ and $F$ is a field. Let $\eta_{i}$ be the nilpotency of $\mathfrak{m}_{i}$. Then $\gamma(A G(R))>1$.

Theorem 4.17 ([19, Theorem 5]). Let $R=R_{1} \times F_{1} \times F_{2} \times \cdots \times F_{m}$ be a commutative ring, where each $\left(R_{1}, \mathfrak{m}_{1}\right)$ is a local ring with $\mathfrak{m}_{1} \neq 0$ and each $F_{j}$ is a field. Let $\eta_{1}$ be the nilpotency of $\mathfrak{m}_{1}$ and $m \geq 3$. Then $\gamma(A G(R))>1$.

Theorem 4.18. Let $R$ be a commutative Artinian ring such that $R=R_{1} \times R_{2} \times \cdots \times R_{n} \times$ $F_{1} \times F_{2} \times \cdots \times F_{m}$, where each $\left(R_{i}, \mathfrak{m}_{i}\right)$ is an Artinian local ring with $\mathfrak{m}_{i} \neq 0$ and each $F_{j}$ is a field. Let $\eta_{i}$ be the nilpotency of $\mathfrak{m}_{i}$ and $n \geq 2$ or $m \geq 3$. Then $\gamma(\mathcal{E} \mathcal{G}(R))>1$.

Proof. Follows from Theorems 4.16 and 4.17.

Theorem 4.19. Let $R$ be a commutative Artinian ring such that $R=R_{1} \times F_{1} \times F_{2}$, where $\left(R_{1}, \mathfrak{m}\right)$ is an Artinian local ring and $F_{1}$ and $F_{2}$ are fields. Let $\eta$ be the nilpotency of $\mathfrak{m}$. Then $\gamma(\mathcal{E G}(R))=1$ if and only if $\eta=2$ and $\mathfrak{m}$ is the only non-trivial ideal of $R_{1}$.

Proof. Suppose that $\eta=2$ and $\mathfrak{m}$ is the only non-trivial ideal of $R_{1}$. Then from Figure 5, we get $\gamma(\mathcal{E} \mathcal{G}(R))=1$, where $a=\mathfrak{m} \times(0) \times(0), b=R_{1} \times(0) \times(0), c=\mathfrak{m} \times F_{1} \times F_{2}$, $d=(0) \times F_{1} \times F_{2}, e=\mathfrak{m} \times(0) \times F_{2}, f=(0) \times F_{1} \times(0), g=R_{1} \times F_{1} \times(0)$, $h=R_{1} \times(0) \times F_{2}, i=(0) \times(0) \times F_{2}, j=\mathfrak{m} \times F_{1} \times(0)$.

Conversely, assume that $\gamma(\mathcal{E} \mathcal{G}(R))=1$. Let $J$ be a non-trivial ideal of $R_{1}$ such that $J \neq \mathfrak{m}$. Then the set $\left\{\mathfrak{m} \times(0) \times(0), \mathfrak{m} \times F_{1} \times(0), J \times F_{1} \times(0),(0) \times F_{1} \times(0)\right\} \cup\{J \times(0) \times$ $\left.(0), \mathfrak{m} \times(0) \times F_{2}, J \times(0) \times F_{2},(0) \times(0) \times F_{2}, R_{1} \times(0) \times(0)\right\}$ induces a copy of $K_{4,5}$ in $\mathcal{E} \mathcal{G}(R)$, which is a contradiction. Hence $\mathfrak{m}$ is the only non-trivial ideal of $R_{1}$.

Theorem 4.20. Let $R$ be a commutative Artinian ring such that $R=R_{1} \times F$, where $\left(R_{1}, \mathfrak{m}\right)$ is an Artinian local ring and $F$ is a field. Let $\eta$ be the nilpotency of $\mathfrak{m}$. Then $\gamma(\mathcal{E G}(R))=1$ if and only if one of the following holds: 


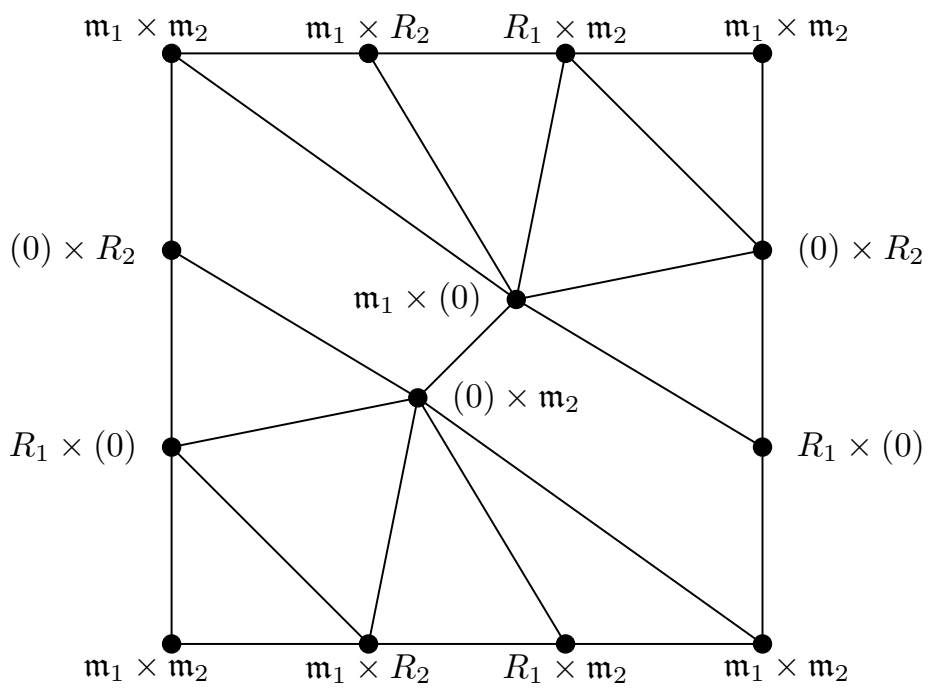

Figure 4: Toroidal embedding of $\mathcal{E} \mathcal{G}\left(R_{1} \times R_{2}\right)$, where $\mathfrak{m}_{i}$ is the only non-trivial ideal of $R_{i}$ for $i=1,2$.

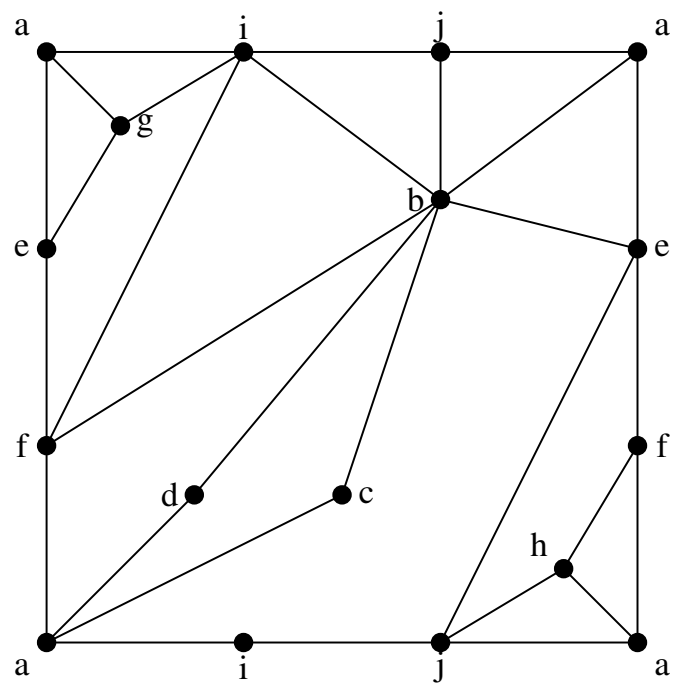

Figure 5: Toroidal embedding of $\mathcal{E} \mathcal{G}\left(R_{1} \times F_{1} \times F_{2}\right)$, where $\mathfrak{m}$ is the only non-trivial ideal of $R_{1}$.

(1) $\eta=3$ and $\mathfrak{m}$ and $\mathfrak{m}^{2}$ are the only non-trivial ideals of $R_{1}$.

(2) $\eta=4$ and $\mathfrak{m}, \mathfrak{m}^{2}$ and $\mathfrak{m}^{3}$ are the only non-trivial ideals of $R_{1}$.

Proof. Suppose that $\gamma(\mathcal{E} \mathcal{G}(R))=1$. If $\eta \geq 5$, then the set $\left\{\mathfrak{m}^{\eta-1} \times(0), \mathfrak{m}^{\eta-2} \times\right.$ $\left.(0), \mathfrak{m}^{\eta-3} \times(0)\right\} \cup\left\{R_{1} \times(0), \mathfrak{m} \times(0),(0) \times F, \mathfrak{m}^{\eta-1} \times F, \mathfrak{m}^{\eta-2} \times F, \mathfrak{m}^{\eta-3} \times F, \mathfrak{m} \times F\right\}$ 


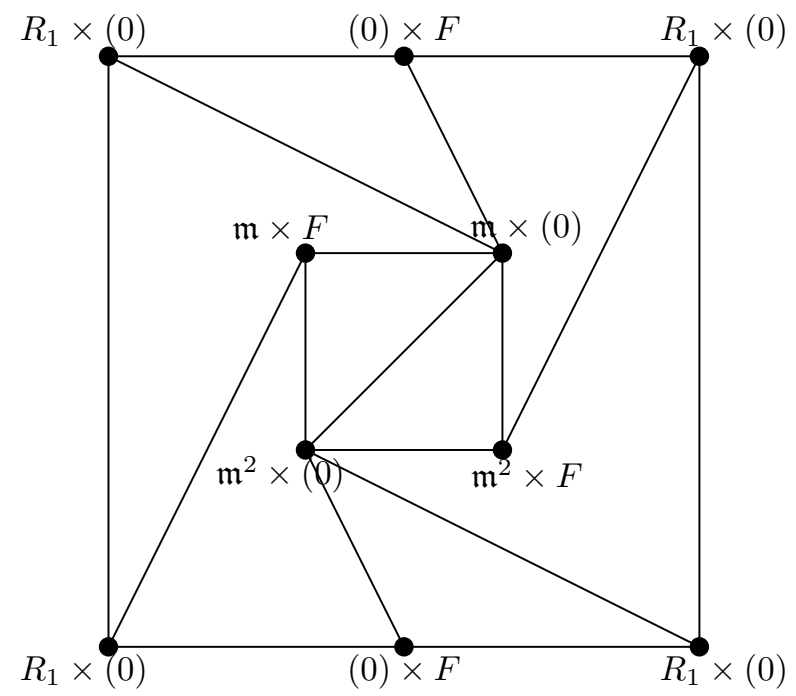

Figure 6: Toroidal embedding of $\mathcal{E} \mathcal{G}\left(R_{1} \times F\right)$, where $\mathfrak{m}$ and $\mathfrak{m}^{2}$ are only non-trivial ideals of $R_{1}$.

induces a copy of $K_{3,7}$. Thus, by Lemma 4.12, $\gamma(\mathcal{E G}(R))>1$, a contradiction. Hence $\eta \leq 4$. We have following cases:

Case I: $\eta=2$. Let $J$ be a non-trivial ideal of $R_{1}$ such that $J \neq \mathfrak{m}$. Then by Lemma 4.7, $R_{1}$ has at least three non-trivial ideals $I_{1}, I_{2}$ and $I_{3}$ such that $I_{1}, I_{2}, I_{3} \neq \mathfrak{m}$. We can see that the set $\left\{R_{1} \times(0), J \times(0), I_{1} \times(0), I_{2} \times(0)\right\} \cup\left\{(0) \times F, J \times F, I_{1} \times F, I_{2} \times F, \mathfrak{m} \times F\right\}$ induces a copy of $K_{3,7}$ in $\mathcal{E} \mathcal{G}(R)$, a contradiction. Hence $\mathfrak{m}$ is the only non-trivial ideal of $R_{1}$. It follows from Theorem 4.10 that $\mathcal{E} \mathcal{G}(R)$ is a planar graph, a contradiction.

Case II: $\eta=3$. Let $I$ be a non-trivial ideal of $R_{1}$ such that $I \neq \mathfrak{m}, \mathfrak{m}^{2}$. Then by Lemma 4.7, $R_{1}$ has at least three non-trivial ideals $I_{1}, I_{2}$ and $I_{3}$ such that $I_{1}, I_{2}, I_{3} \neq \mathfrak{m}, \mathfrak{m}^{2}$. It is easy to see that the set $\left\{R_{1} \times(0), \mathfrak{m} \times(0), \mathfrak{m}^{2} \times(0)\right\} \cup\left\{I \times(0), I_{1} \times(0), I_{2} \times(0), 0 \times F\right.$, $\left.\mathfrak{m} \times F, \mathfrak{m}^{2} \times F, I \times F\right\}$ induces a copy of $K_{3,7}$ in $\mathcal{E} \mathcal{G}(R)$, a contradiction. Hence $\mathfrak{m}$ and $\mathfrak{m}^{2}$ are the only non-trivial ideals of $R_{1}$.

Case III: $\eta=4$. Let $I$ be a non-trivial ideal of $R_{1}$ such that $I \neq \mathfrak{m}^{i}$ for each $i=1,2,3$. Then by Lemma 4.7, $R_{1}$ has at least three non-trivial ideals $I_{1}, I_{2}$ and $I_{3}$ such that $I_{1}, I_{2}, I_{3} \neq \mathfrak{m}^{i}$ for each $i=1,2,3$. It is easy to see that the set $\{\mathfrak{m} \times(0)$, $\left.\mathfrak{m}^{2} \times(0), \mathfrak{m}^{3} \times(0)\right\} \cup\left\{R_{1} \times(0), I \times(0), I_{1} \times(0), I_{2} \times(0), \mathfrak{m} \times F, \mathfrak{m}^{2} \times(0), \mathfrak{m}^{3} \times(0)\right\}$ induces a copy of $K_{3,7}$ in $\mathcal{E} \mathcal{G}(R)$, a contradiction. Hence $\mathfrak{m}, \mathfrak{m}^{2}$ and $\mathfrak{m}^{3}$ are the only nontrivial ideals of $R_{1}$.

Conversely, if $\mathfrak{m}$ and $\mathfrak{m}^{2}$ are the only non-trivial ideals of $R_{1}$, then $\left|A^{*}(R)\right|=6$ and the set $\left\{R_{1} \times(0), \mathfrak{m} \times(0), \mathfrak{m}^{2} \times(0)\right\} \cup\left\{(0) \times F, \mathfrak{m} \times F, \mathfrak{m}^{2} \times F\right\}$ induces a copy of $K_{3,3}$ in $\mathcal{E} \mathcal{G}(R)$. Thus, $K_{3,3} \leq \mathcal{E} \mathcal{G}(R) \leq K_{6}$, which implies that $\gamma(\mathcal{E} \mathcal{G}(R))=1$.

Now, if $\mathfrak{m}, \mathfrak{m}^{2}$ and $\mathfrak{m}^{3}$ are the only non-trivial ideals of $R_{1}$. Then from Figure 7 , $\gamma(\mathcal{E} \mathcal{G}(R))=1$. 


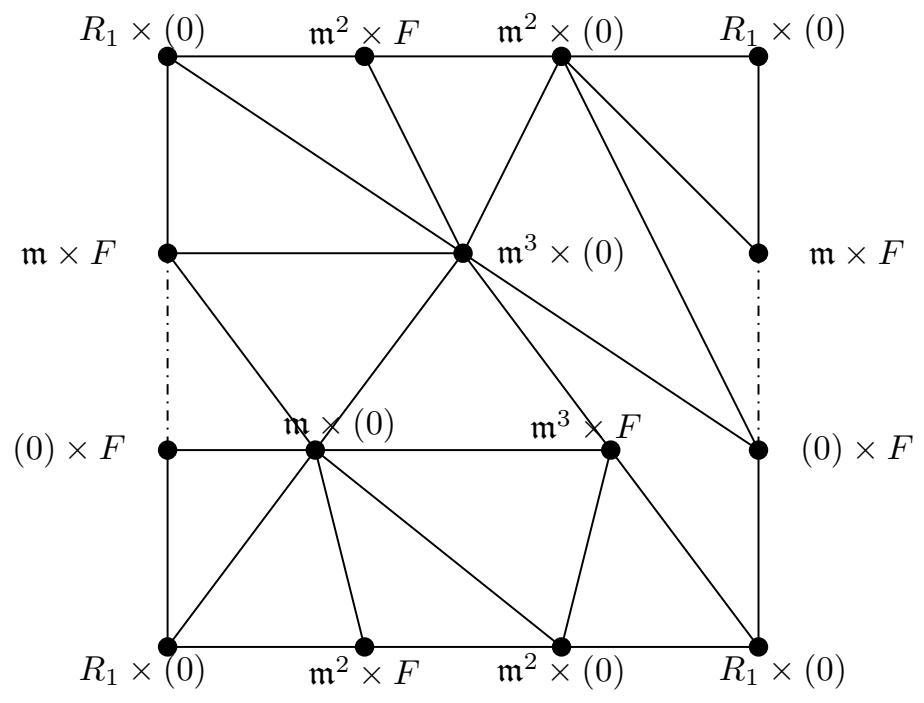

Figure 7: Toroidal embedding of $\mathcal{E} \mathcal{G}\left(R_{1} \times F\right)$, where $\mathfrak{m}, \mathfrak{m}^{2}$ and $\mathfrak{m}^{3}$ are non-trivial ideals of $R_{1}$.

\section{ORCID iDs}

Mohd Nazim (iD https://orcid.org/0000-0001-8817-4336

Nadeem ur Rehman (D) https://orcid.org/0000-0003-3955-7941

\section{References}

[1] G. Aalipour, S. Akbari, M. Behboodi, R. Nikandish, M. J. Nikmehr and F. Shaveisi, The classification of the annihilating-ideal graphs of commutative rings, Algebra Colloq. 21 (2014), 249-256, doi:10.1142/s1005386714000200.

[2] G. Aalipour, S. Akbari, R. Nikandish, M. Nikmehr and F. Shaveisi, On the coloring of the annihilating-ideal graph of a commutative ring, Discrete Math. 312 (2012), 2620-2626, doi: 10.1016/j.disc.2011.10.020.

[3] G. Aalipour, S. Akbari, R. Nikandish, M. J. Nikmehr and F. Shaveisi, Minimal prime ideals and cycles in annihilating-ideal graphs, Rocky Mountain J. Math. 43 (2013), 1415-1425, doi: 10.1216/rmj-2013-43-5-1415.

[4] M. Ahrari, S. A. S. Sabet and B. Amini, On the girth of the annihilating-ideal graph of a commutative ring, J. Linear Topol. Algebra 4 (2015), 209-216.

[5] S. Akbari and A. Mohammadian, On the zero-divisor graph of a commutative ring, J. Algebra 274 (2004), 847-855, doi:10.1016/s0021-8693(03)00435-6.

[6] F. Aliniaeifard, M. Behboodi, E. Mehdi-Nezhad and A. M. Rahimi, On the diameter and girth of an annihilating-ideal graph, 2014, arXiv:1411.4163v1 [math.CO].

[7] D. D. Anderson and M. Naseer, Beck's coloring of a commutative ring, J. Algebra 159 (1993), 500-514, doi:10.1006/jabr.1993.1171.

[8] D. F. Anderson and P. S. Livingston, The zero-divisor graph of a commutative ring, J. Algebra 217 (1999), 434-447, doi:10.1006/jabr.1998.7840. 
[9] M. F. Atiyah and I. G. Macdonald, Introduction to Commutative Algebra, Addison-Wesley Publishing Co., Reading, Mass.-London-Don Mills, Ont., 1969.

[10] I. Beck, Coloring of commutative rings, J. Algebra 116 (1988), 208-226, doi:10.1016/ 0021-8693(88)90202-5.

[11] M. Behboodi and Z. Rakeei, The annihilating-ideal graph of commutative rings I, J. Algebra Appl. 10 (2011), 727-739, doi:10.1142/s0219498811004896.

[12] M. Behboodi and Z. Rakeei, The annihilating-ideal graph of commutative rings II, J. Algebra Appl. 10 (2011), 741-753, doi:10.1142/s0219498811004902.

[13] F. R. DeMeyer, T. McKenzie and K. Schneider, The zero-divisor graph of a commutative semigroup, Semigroup Forum 65 (2002), 206-214, doi:10.1007/s002330010128.

[14] D. Dolžan and P. Oblak, The zero-divisor graphs of rings and semirings, Internat. J. Algebra Comput. 22 (2012), 1250033, 20, doi:10.1142/s0218196712500336.

[15] T. Y. Lam, A First Course in Non-Commutative Rings, Springer-Verlag, New York, 1991, doi: 10.1007/978-1-4684-0406-7.

[16] M. J. Nikmehr and S. M. Hosseini, More on the annihilator-ideal graph of a commutative ring, J. Algebra Appl. 18 (2019), 1950160, 14, doi:10.1142/s0219498819501603.

[17] M. J. Nikmehr, R. Nikandish and M. Bakhtyiari, On the essential graph of a commutative ring, J. Algebra Appl. 16 (2017), 1750132, 14, doi:10.1142/s0219498817501328.

[18] S. P. Redmond, The zero-divisor graph of a non-commutative ring, in: Internat. J. Commutative Rings, Nova Sci. Publ., Hauppauge, NY, volume 1, 2002.

[19] K. Selvakumar and P. Subbulakshmi, Classification of rings with toroidal annihilating-ideal graph, Commun. Comb. Optim. 3 (2018), 93-119, doi:10.22049/cco.2018.26060.1072.

[20] K. Selvakumar, P. Subbulakshmi and J. Amjadi, On the genus of the graph associated to a commutative ring, Discrete Math. Algorithms Appl. 9 (2017), 1750058, 11, doi:10.1142/ s1793830917500586.

[21] D. B. West, Introduction to Graph Theory, Prentice-Hall of India, New Delhi, 2002.

[22] A. T. White, Graphs, Groups and Surfaces, North-Holland Mathematics Studies, No. 8, NorthHolland Publishing Co., Amsterdam, 1973. 